\title{
Reduced inspection time in active thermographic non-destructive testing of low- thermal-conductivity materials
}

\author{
by M. Ishikawa*, M. Koyama**, H. Kasano***, H. Hatta**** S. Utsunomiya**** \\ * Tokushima University, Graduate School of Technology, Industrial and Social Sciences, 2-1 Minamijousanjima- \\ cho, 770-8506, Tokushima, Japan, m.ishikawa@tokushima-u.ac.jp \\ ${ }^{* *}$ Meisei University, School of Science and Engineering, 2-1-1 Hodokubo, Hino, Tokyo, 191-8506, Japan, \\ masashi.koyama@meisei-u.ac.jp \\ ${ }^{* * \star}$ Nihon University, Department of Civil Engineering, Nakagawara, Tokusada, Tamura, Koriyama, Fukushima, \\ 963-8642, Japan, kasano@civil.ce.nihon-u.ac.jp \\ **** Japan Aerospace Exploration Agency, 3-1-1 Yoshinodai, Chuo-ku, Sagamihara, Kanagawa, 252-5210, \\ Japan,hatta.hiroshi@jaxa.jp
}

\begin{abstract}
Inspecting low-thermal-conductivity materials via active thermographic non-destructive testing requires a long inspection time because of the slow heat transfer in such materials. This study focuses on reducing the inspection time by using thermal waves with a high-frequency component. Fourier transform was applied to extract the high-frequency component from the observed thermal data. Consequently, it was observed from analytical studies and experiments of a carbon-fiber-reinforced plastic and a concrete specimen that using phase data in the high-frequency range obtained from the transformed data is effective for the detection of internal defects within a short inspection time.
\end{abstract}

\section{Introduction}

Active thermographic inspection is a convenient non-destructive testing method; it can be used to inspect a relatively large area in a single test without physical contact with the test object. However, the active thermographic inspection of low-thermal-conductivity materials requires a long inspection time because heat transfer in such materials is slow. This is a practical problem associated with the inspection of polymer materials or concrete structures.

In order to reduce the inspection time of the active thermographic method, we focused on using the relationship between the heat propagation speed and frequency of the propagating thermal wave. According to the thermal wave theory, thermal waves with a high-frequency component propagate faster $[1,2]$. Thus, using heat with a high-frequency component is expected to be effective in reducing the inspection time. In order to extract the high-frequency component from the observed thermal data, we used phase data obtained by applying Fourier transform to the thermal data. The phase transformation technique is known as pulsed phase thermography (PPT), wherein instantaneous pulse heating is used as the heat-excitation method [2,3]. By applying the Fourier transform, phase data at an arbitrary frequency can be extracted. In addition, many studies have reported that PPT is effective for enhancing the detectable defect depth and suppressing the non-uniform heating effect $[3,4,5,6]$. These advantages are expected to be effective for detecting a small signal observed in the high-frequency range because thermal waves with higher-frequency components are damped more significantly.

In this study, the effect of the aforementioned method is examined through analytical studies and experiments with a carbon-fiber-reinforced plastic (CFRP) and a concrete specimen. The thermal diffusivity of CFRP laminates in the through thickness direction is generally very small because it is nearly equal to that of the resin matrix. The thermal diffusivity of concretes is also small; in addition, inspection of concretes requires a long time because defects in concretes are frequently located several tens of millimeters from the surface. In this report, first, inspections using instantaneous flash heating (i.e., PPT method) are discussed in the following section. The effect of inspection time reduction is estimated though analytical studies and verified though experiments, Second, inspections using longduration heating and phase data are discussed in Section 3. Long-duration heating can input larger amount of heat than the flash heating method; thus, it is expected to be effective for the inspection of such concretes. We examined the extraction of high-frequency components in the heat input provided by long-duration heating by using the phase transformation technique, and investigated the capability of defect detection and effect of inspection time reduction.

\section{Inspections using instantaneous flash heating}

\subsection{Analytical studies}

In order to examine the effect of inspection time reduction, analytical studies using a one-dimensional plate model were performed. In the analyses, a plate with the thickness of $H$ was considered $(H$ was assumed to be the depth 
of an ideal defect with infinite in-plane size). When Dirac pulse heating was applied to the surface, the temperature-time data of the same surface were calculated using the following equation [5]:

$$
T(t)=\frac{2 Q}{\rho c H} \sum_{n=1}^{\infty} \frac{\mu_{\mathrm{n}}^{2}}{B_{\mathrm{i}}^{2}+2 B_{\mathrm{i}}+\mu_{\mathrm{n}}^{2}} \mathrm{e}^{-\mu_{\mathrm{n}}^{2} F_{\mathrm{O}}},
$$

where $\rho$ and $c$ are the density and specific heat, respectively. $B_{\mathrm{i}}$ denotes the Biot number and is defined as $B_{\mathrm{i}}=h H / k$, where $h$ and $k$ are the surface heat exchange coefficient and thermal conductivity, respectively, and Fo is Fourier number defined as $F_{0}=\alpha t / H^{2}$, in which $\alpha$ represents thermal diffusivity. $\mu_{\mathrm{n}}$ is determined from the following relation:

$$
\tan (\mu)=\frac{2 \frac{\mu h}{H k}}{\left(\frac{\mu}{H}\right)^{2}-\left(\frac{h}{k}\right)^{2}} .
$$

Subsequently, the Fourier transform was applied to Eq. (1) in order to obtain the phase-frequency data, which were obtained as

$$
F(f)=\frac{2 Q}{\rho c H} \sum_{\mathrm{n}=1}^{\infty} \frac{\mu_{\mathrm{n}}^{2}}{B_{\mathrm{i}}^{2}+2 B_{\mathrm{i}}+\mu_{\mathrm{n}}^{2}} \frac{1}{\frac{\mu_{\mathrm{n}}^{2} \alpha}{H^{2}}+j 2 \pi f},
$$

where $f$ is the frequency and $j$ denotes an imaginary unit. By assigning the real and imaginary parts $(R(f)$ and $I(f))$ in Eq. (3) to the following equation, the phase-frequency $\varphi(f)$ after instantaneous pulse heating can be obtained.

$$
\varphi(f)=\tan ^{-1} \frac{l(f)}{R(f)}
$$

Figure 1 shows the calculated phase-frequency data of the material properties of CFRP (density $\rho=1638$ $\mathrm{kg} / \mathrm{m}^{3}$, specific heat $c=870 \mathrm{~J} /(\mathrm{kg} \mathrm{K})$, and thermal conductivity $\left.k=0.56 \mathrm{~W} /(\mathrm{m} \mathrm{K})\right)$. It is observed from Fig. 1 that $\varphi(f)$ has a local minimum peak $P_{\mathrm{L}}$ for each $H$ condition and that the frequency at $P_{\mathrm{L}}$ decreases with the increase in $H$. In addition to $P_{\mathrm{L}}$ in the low-frequency range, the phase shows a small local maximum peak $\left(P_{H}\right)$ in the high-frequency range. Thus, using the phase images at the frequency of $P_{H}$ should be effective not only to detect defects but also to reduce inspection time. Figure 2 shows the relationship between the frequencies at $P_{\mathrm{L}}$ and $P_{\mathrm{H}}$ (denoted as $\hbar_{\mathrm{L}}$ and $f_{\mathrm{H}}$, respectively) and $H$. For example, when $H=4 \mathrm{~mm}, \hbar_{\mathrm{L}}$ and $f_{H}$ are 0.0027 and $0.03 \mathrm{~Hz}$, respectively. The minimum frequency obtained using Fourier transform depends on the thermal data acquisition time (i.e., inspection time $t_{\text {data) }}$, and is equal to $1 / t_{\text {data. }}$. Therefore, although a period of $370 \mathrm{~s}$ is required to obtain the phase at $\mathrm{t}$, the inspection time could be reduced to $33 \mathrm{~s}$ when the phase at $f_{H}$ is used.

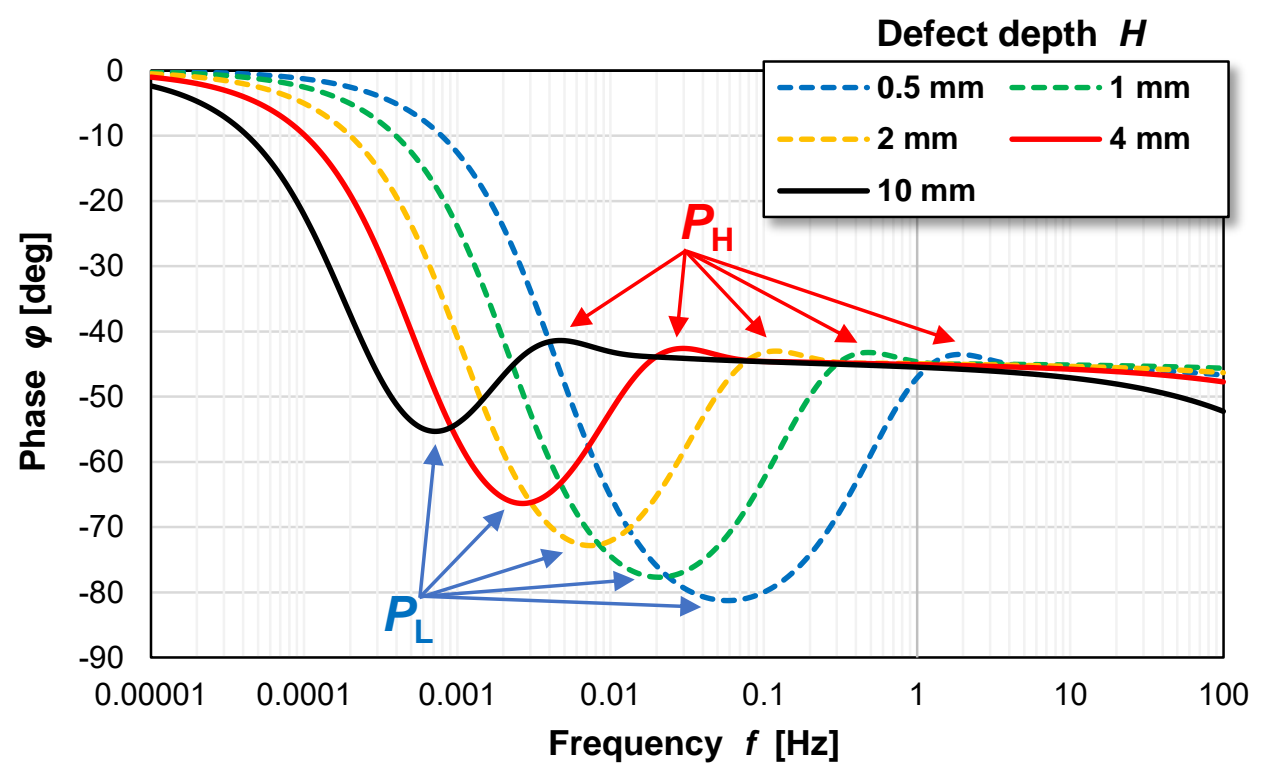

Fig. 1. Phase-frequency data determined from $1 D$ analytical calculations of the material properties of CFRP. 


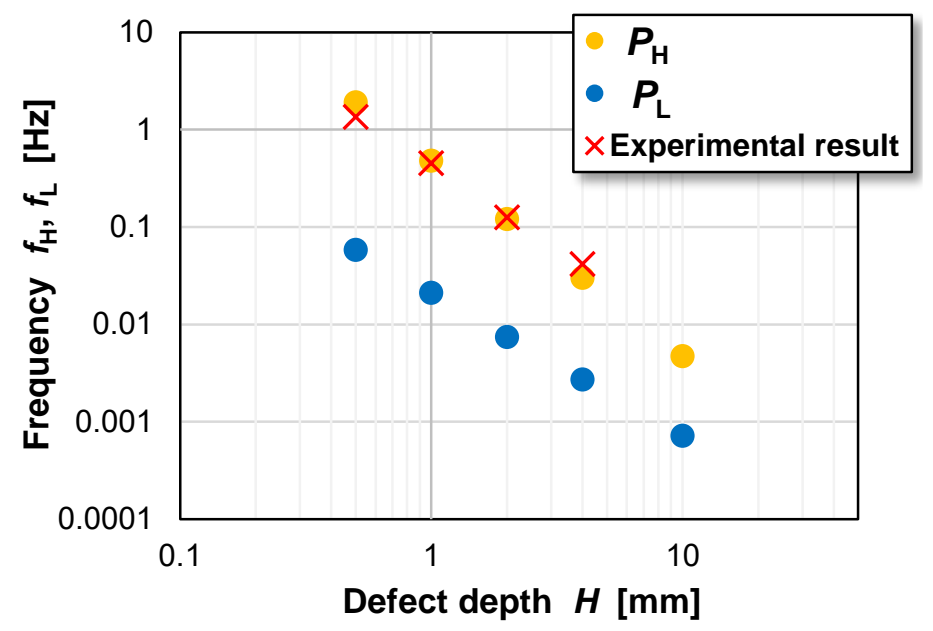

Fig. 2. Relationship between the frequencies at $P_{L}$ and $P_{H}\left(f_{L}\right.$ and $f_{H}$, respectively) and defect depth $H$.

\subsection{Experiments} were performed.

In order to verify the effect of reduction of inspection time, experiments with a CFRP and a concrete specimen

\subsubsection{Experimental setup}

Figures 3 and 4 show the CFRP and concrete specimen used in the experiments, respectively. The CFRP specimen, of dimensions $200 \times 200 \times 20 \mathrm{~mm}$, has some flat-bottomed holes as artificial defects. The defect diameter was $20 \mathrm{~mm}$, and its depth from the heated surface varied from $0.5 \mathrm{~mm}$ to $15 \mathrm{~mm}$. The concrete specimen has dimensions $300 \times 300 \times 100 \mathrm{~mm}$, with a slit defect (2-mm-thick air-gap) at a depth of $10 \mathrm{~mm}$ from the surface. The specimens were heated using two xenon flash lamps (the output of each was $1000 \mathrm{~J}$ ), and the surface temperature after heating was monitored using an infrared camera (A315, FLIR Systems, Inc.). Subsequently, the obtained temperaturetime data were transformed into phase-frequency data by applying Fourier transform.

\subsubsection{Results}

The phase images obtained from the experiments with the CFRP specimen are presented in Fig. 5. The defects are detected at the frequency of $f_{\mathrm{L}}$ for each depth. Moreover, the defects are identified in the phase images at $f_{H}$ with a phase contrast inversion (i.e., phase in the defective area is larger than that in the surrounding area when using $f_{\mathrm{H}}$, although it is smaller than that in the surrounding area when using $f_{\mathrm{L}}$ ). These results show that using phase data at $f_{\mathrm{H}}$ is also effective for detecting defects. With regard to the inspection time, for example, the $\mathrm{f}$ for a 2-mm-deep defect was

(a)

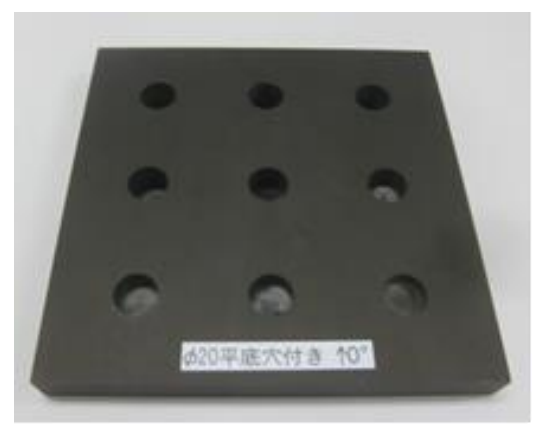

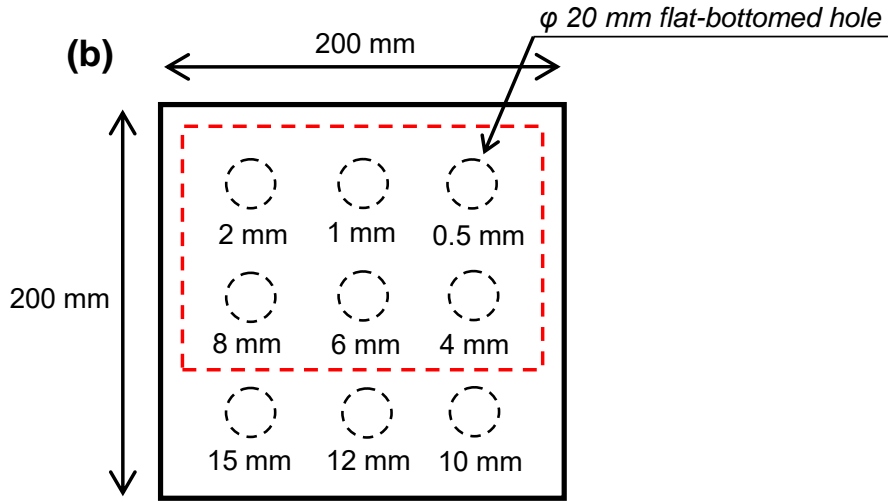

Fig. 3. CFRP specimen with flat-bottomed holes as artificial defects: (a) photograph, and (b) schematic configuration. 
(a)

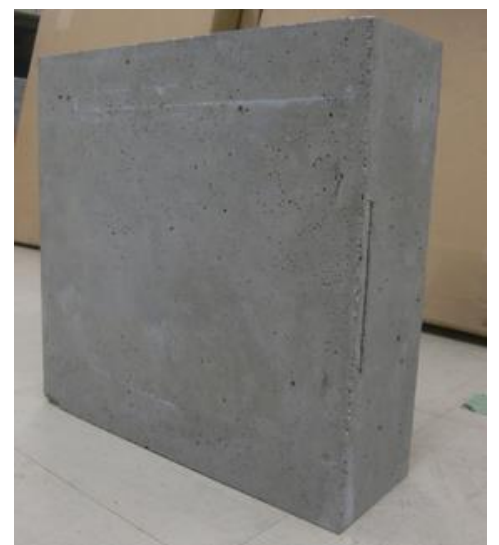

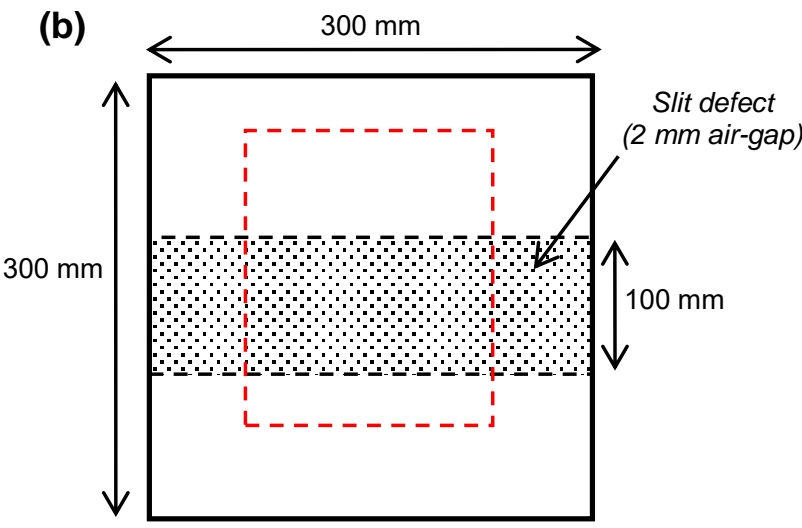

Fig. 4. Concrete specimen with a slit defect: (a) photograph, and (b) schematic configuration.

$0.0074 \mathrm{~Hz}$; thus, the required inspection time was $135 \mathrm{~s}$. In contrast, the $f_{\mathrm{H}}$ for a 2-mm-deep defect was $0.12 \mathrm{~Hz}$, and the required inspection time was reduced to $8.3 \mathrm{~s}$. The experimentally obtained $f_{H}$ values (determined as the frequency at the peak of the fourth-order approximation curve for the phase data around the local maximum) are compared with the estimated frequencies calculated in the analytical studies in Fig. 2. The experimental results are consistent with the analytical results. These results verify the validity of the analytical results, and suggest the effectiveness of using phase data at $f_{\mathrm{H}}$ for reducing the inspection time.

The obtained phase images for the concrete specimen are presented in Fig. 6. From the analytical calculation of the material properties of concrete (density $\rho=2240 \mathrm{~kg} / \mathrm{m}^{3}$, specific heat $c=800 \mathrm{~J} /(\mathrm{kg} \mathrm{K})$, and thermal conductivity $k=$ $1.7 \mathrm{~W} /(\mathrm{m} \mathrm{K})), f_{\mathrm{L}}$ and $f_{\mathrm{H}}$ for a $10-\mathrm{mm}$-deep defect in concrete are estimated as 0.001 and $0.012 \mathrm{~Hz}$, respectively. It is observed from Fig. 6 that the slit area can be identified not only in the phase image at $f_{\mathrm{L}}$ but also in the phase image at $f_{\mathrm{H}}$

(a)

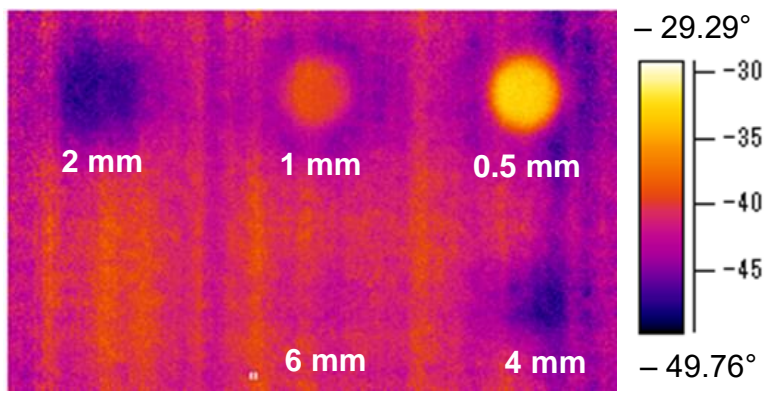

(c)
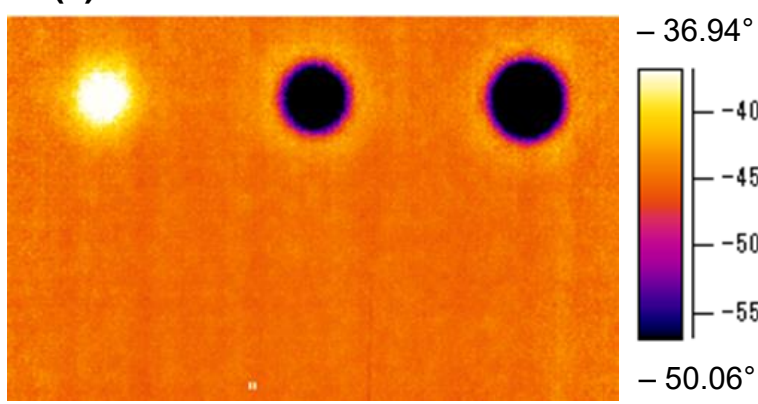

(b)

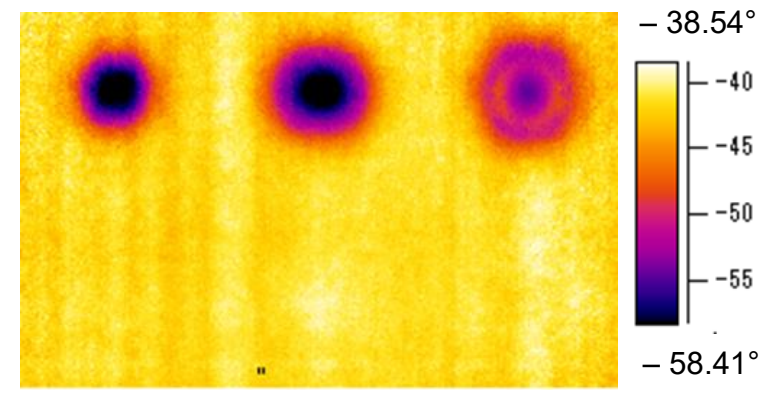

(d)

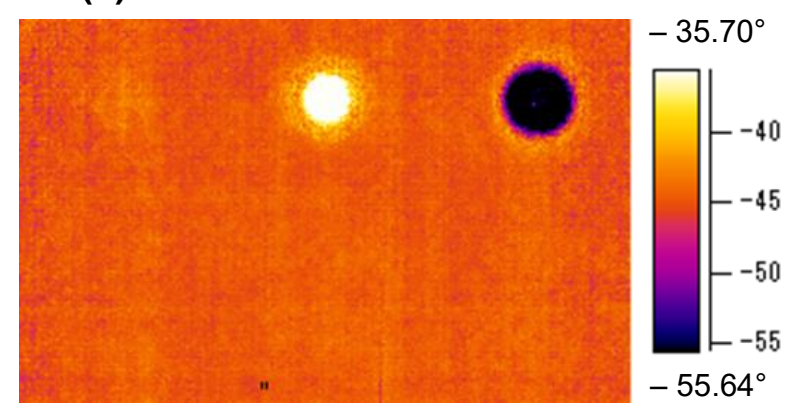

Fig. 5. Phase images obtained from the experiments with a CFRP specimen (area enclosed by red dashed line in Fig. 3(b)) at the frequencies of (a) $0.0074 \mathrm{~Hz}$ (fL for $\mathrm{H}=2 \mathrm{~mm}$ ), (b) $0.021 \mathrm{~Hz}$ (f f for $\mathrm{H}=1 \mathrm{~mm}$ ), (c) $0.12 \mathrm{~Hz}$ (fH for $\mathrm{H}=2 \mathrm{~mm})$, and (d) $0.42 \mathrm{~Hz}\left(f_{H}\right.$ for $\left.\mathrm{H}=1 \mathrm{~mm}\right)$. The numerals in the figures denote defect depth. 
(a)

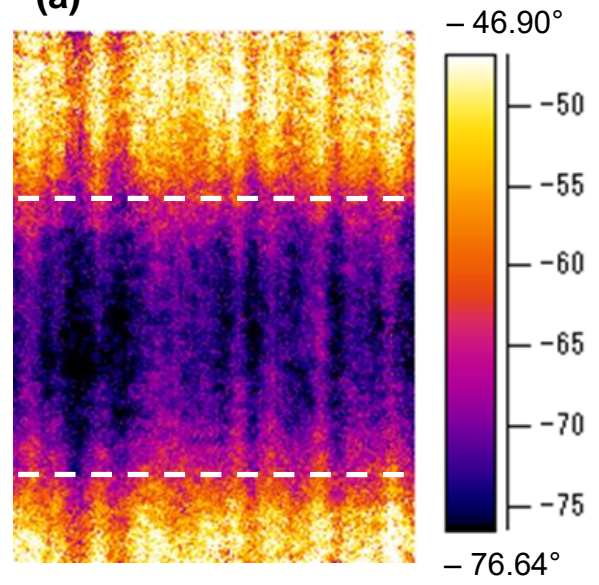

(b)

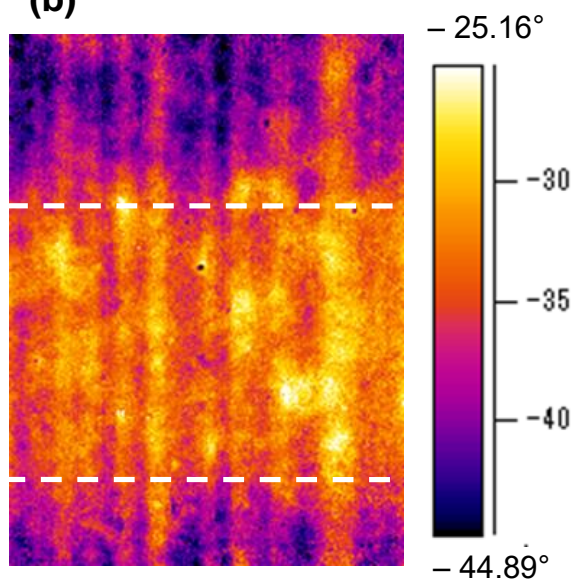

Fig. 6. Phase images obtained from the experiments with a concrete specimen with a 10-mm-deep slit defect (area enclosed by the red dashed line in Fig. 4(b)) at the frequencies of (a) $0.001 \mathrm{~Hz}$ ( $f_{L}$ for $\mathrm{H}=10 \mathrm{~mm}$ ) and (b) $0.012 \mathrm{~Hz}\left(f_{H}\right.$ for $H=10 \mathrm{~mm}$ ). The area enclosed by the dashed lines indicates the defective area.

\section{Inspections using long-duration heating}

When inspecting thick concretes, a large heat input is required. In long-duration heating, heat input can be increased easily by adjusting heating duration. The heat input provided by long-duration heating also includes a wide range of frequency components, although the frequency range is narrower than that of the input provided by flash heating. Thus, by extracting the higher-frequency component of the input heat, inspections could be performed in shorter time.

In order to examine the above prediction, experiments were performed using long-duration heating. In the experiments, the same concrete specimen used in the previous section was employed. The specimen was heated using a $500 \mathrm{~W}$ halogen lamp for 100,200 , and $300 \mathrm{~s}$, and the surface temperature during heating was monitored using the infrared camera (thus, the inspection time was equal to the heating duration). The obtained temperature-time data were transformed into phase-frequency data by applying Fourier transform.

Figure 7 shows the experimentally obtained phase images at the frequencies of $0.0033,0.005$, and $0.01 \mathrm{~Hz}$; they were obtained from the experiments for heating durations of 300,200 , and $100 \mathrm{~s}$, respectively. It is observed from Fig. 7 that the slit defect is identified in all the phase images, even in the image corresponding to the frequency of 0.01 $\mathrm{Hz}$. With regard to the thermal diffusion length, $\lambda(=\sqrt{\alpha / \pi f})$, the frequency of the thermal wave required to propagate 20 $\mathrm{mm}$ in concretes (round-trip distance to a 10-mm-deep defect) is estimated as $0.0008 \mathrm{~Hz}$. Therefore, the experimental results demonstrated that defects could be detected in the phase images at frequencies higher than that estimated from

(a)

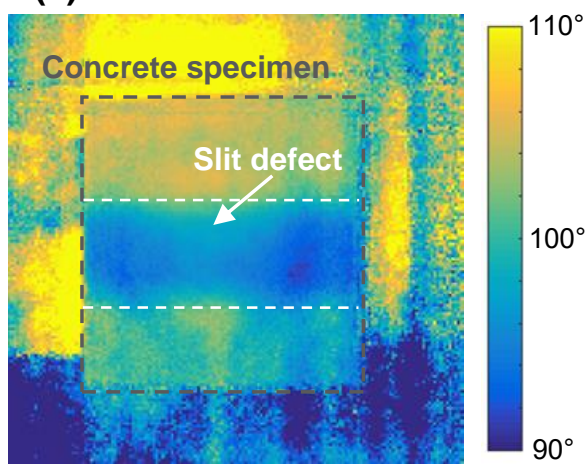

(b)

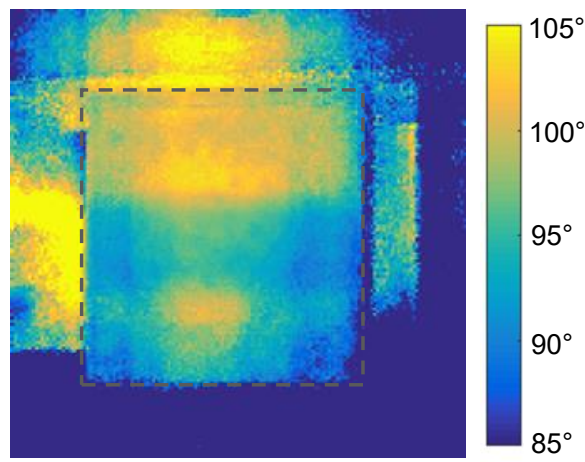

(c)

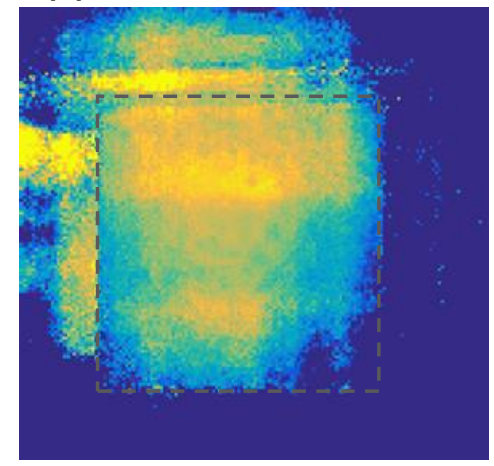

[deg]

$\square^{105^{\circ}}$

$100^{\circ}$

$95^{\circ}$

$90^{\circ}$

$85^{\circ}$

Fig. 7. Phase images of the concrete specimen obtained from the experiments using long-duration heating at the frequencies of (a) $0.0033 \mathrm{~Hz}$, (b) $0.005 \mathrm{~Hz}$, and (c) $0.01 \mathrm{~Hz}$. 
the thermal diffusion length theory. The inspection time required to obtained the phase image at $0.01 \mathrm{~Hz}(100 \mathrm{~s})$ is similar to that obtained by using phase data at $f_{H}$ obtained after instantaneous flash heating ( $f_{H}$ for 10 -mm-thick concrete is $0.012 \mathrm{~Hz}$; thus the required inspection time is approximately $83 \mathrm{~s})$. With regard to input frequency component, Fig. 8(a) shows the observed temperature on the non-defective surface for each heating duration, and Fig. 8(b) shows the spectrum amplitude as a function of frequency calculated by applying Fourier transform to the data in Fig. 8(a). In Fig. 8, the results obtained from the experiment using flash heating are also presented for comparison. It is observed from Fig. 8(b) that the spectrum amplitude of the input heat of long-duration heating is larger than that of flash heating when the frequency is lower than approximately $0.1 \mathrm{~Hz}$. Therefore, in the present experiments, the inspection capability achieved while using long-duration heating should be higher than that achieved while using flash lamp heating when phase data at approximately $0.01 \mathrm{~Hz}$ is used. These results suggest that using phase data in the high-frequency range obtained by applying long-duration heating is also an effective way to reduce the inspection time.
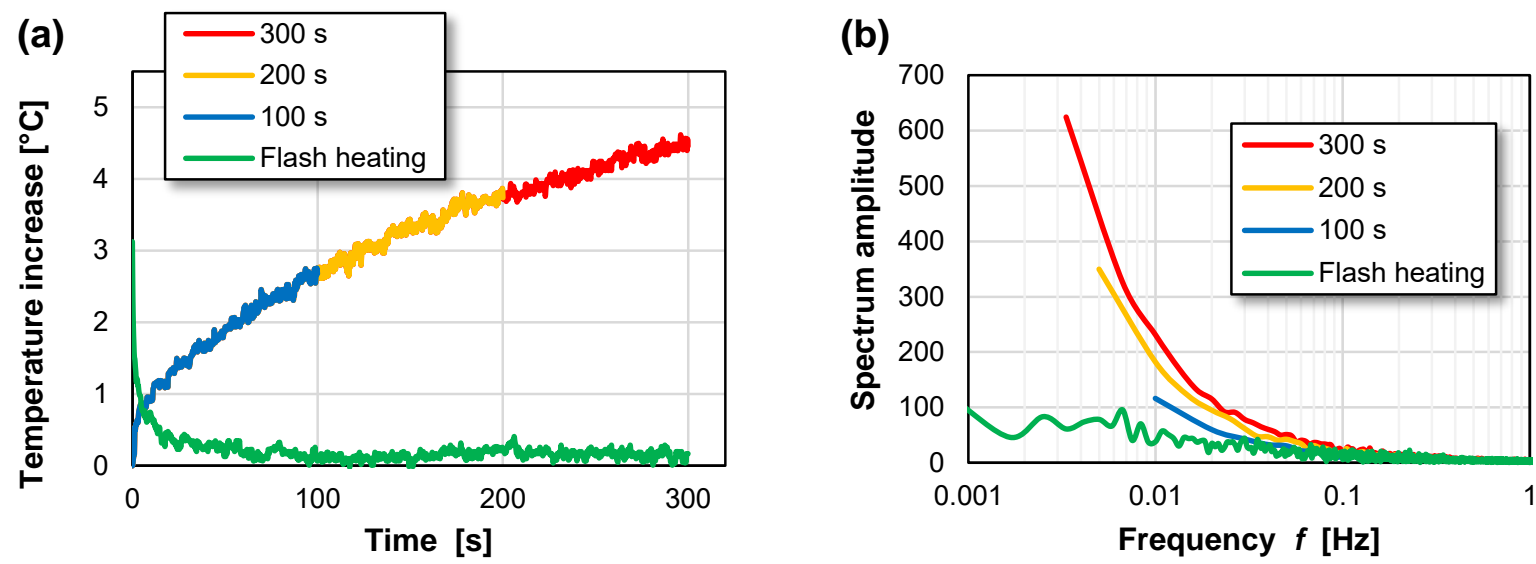

Fig. 8. (a) Experimentally observed thermal data on the non-defective area when applying long-duration heating (100, 200, and 300 s) and flash heating, (b) spectrum amplitude as a function of frequency calculated by applying Fourier transform to the thermal data in Fig. 8(a).

\section{Conclusions}

Methods for reducing inspection time in active thermography by using a high-frequency thermal component and phase transformation technique were examined in this study. In instantaneous flash heating, using phase data at a small local maximum peak observed in the high-frequency region was effective for detecting defects within a short inspection time. In the inspections using long-duration heating, a defect was detected in the phase images at frequencies higher than that estimated considering the thermal diffusion length. These results suggest that extracting a high-frequency component of input heat by applying Fourier transform and inspection using phase data in the high-frequency region is an effective method for reducing the inspection time of active thermographic non-destructive testing, especially when inspecting low-thermal-conductivity materials.

\section{Acknowledgements}

This work was supported by JSPS KAKENHI Grant Numbers 17K01296, 26282099.

\section{REFERENCES}

[1] Carslaw H.S., Jaeger J.C., "Conduction of heat in solids". Oxford University Press, Oxford, 1959.

[2] Maldague X., "Theory and practice of infrared technology for nondestructive testing". John Wiley \& Sons, New York, 2001.

[3] Maldague X., Marinetti S., Pulse phase infrared thermography, J. appl. Phys. - Vol. 79, no 5, pp. 2694-2698, 1996.

[4] Weritz F., Arndt R., Röllig M., Maierhofer C., Wiggenhauser H., Investigation of concrete structures with pulse phase thermography, Materials and Structures. - Vol. 38, no 9, pp. 843-849, 2005.

[5] Montanini R., Quantitative determination of subsurface defects in a reference specimen made of Plexiglas by means of lock-in and pulse phase infrared thermography, Infrared Phys. Technol. - Vol. 53, no 5, pp. 363-371, 2010.

[6] Ishikawa M., Hatta H., Habuka Y., et al., Detecting deeper defects using pulse phase thermography. Infrared Phys. Technol. - Vol. 57, pp. 42-49, 2013. 